\title{
GRB Afterglows and Other Transients in the SDSS
}

Brian C. Lee*, Daniel E. Vanden Berk*, Don Lamb ${ }^{\dagger}$, Brian Wilhite ${ }^{\dagger}$, Daniel E.

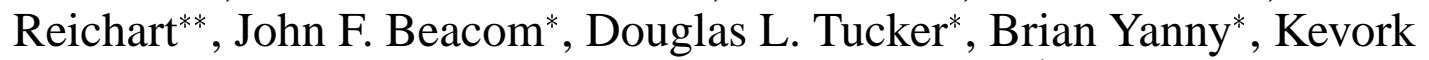
Abazajian*, Jennifer Adelman*, James Annis*, Bing Chen ${ }^{\ddagger}$, Mike Harvanek ${ }^{\S}$, Arne Henden $^{\mathbb{I}}$, Kevin Hurley ${ }^{\|}$, Zeljko Ivezic ${ }^{\dagger \dagger}$, Robert Kehoe ${ }^{\ddagger \ddagger}$, Scot Kleinman ${ }^{\S}$, Richard Kron*, Jurek Krzesinski ${ }^{\S}$, Dan Long ${ }^{\S}$, Timothy McKay ${ }^{\S \S}$, Russet McMillan ${ }^{\S}$, Eric H. Neilsen*, Peter R. Newman ${ }^{\S}$, Atsuko Nitta ${ }^{\S}$, Povilas Palunas ${ }^{\text {IfII }}$, Donald P. Schneider $^{* * *}$, Steph Snedden ${ }^{\S}$, James Wren ${ }^{\dagger \dagger}$, Don York ${ }^{\dagger}$, John W. Briggs ${ }^{\dagger+}$, J. Brinkmann $^{\S}$, Istvan Csabai ${ }^{\ddagger}$, Greg S. Hennessy ${ }^{\S \S}$, Stephen Kent*, Robert Lupton ${ }^{\dagger \dagger}$, Heidi Jo Newberg ${ }^{\text {IIIIII }}$ and Chris Stoughton*

\footnotetext{
* Experimental Astrophysics Group, Fermi National Accelerator Laboratory, P.O. Box 500, Batavia, IL 60510

${ }^{\dagger}$ Department of Astronomy and Astrophysics, University of Chicago, 5640 South Ellis Avenue, Chicago, IL 60637

${ }^{* *}$ Palomar Observatory, 105-24, California Institute of Technology, Pasadena, CA 91125

${ }^{\ddagger}$ Department of Physics and Astronomy, Johns Hopkins University, 3701 San Martin Drive, Baltimore, MD 21218

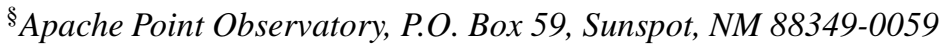

II Universities Space Research Association / U. S. Naval Observatory, Flagstaff Station, P. O. Box 1149, Flagstaff, AZ 86002-1149

"University of California at Berkeley, Space Science Laboratory, Grizzly Peak and Centennial Drive, Berkeley, CA 94720

${ }^{\dagger}$ Princeton University Observatory, Peyton Hall, Princeton, NJ 08544-1001

*Michigan State University, Department of Physics and Astronomy, East Lansing, Michigan 48824

${ }^{\S}$ University of Michigan, Department of Physics, 500 East University, Ann Arbor, MI 48109

${ }^{I I I}$ Catholic University of America, NASA/Goddard Space Flight Center, Code 681, Greenbelt, MD 20771

*** Astronomy and Astrophysics Department, Pennsylvania State University, 525 Davey Laboratory, University Park, PA 16802

${ }^{\dagger \dagger}$ Los Alamos National Laboratory, PO Box 1663, Los Alamos, NM 87545

\#+Yerkes Observatory, University of Chicago, 373 West Geneva Street, Williams Bay, WI 53191

$\S_{\S}$ U.S. Naval Observatory, 3450 Massachusetts Ave., NW, Washington, DC 20392-5420

IIIII Physics Department, Rensselaer Polytechnic Institute, SC1C25, Troy, NY 12180
}

\begin{abstract}
The Sloan Digital Sky Survey (SDSS) will image one quarter of the sky centered on the northern galactic cap and produce a 3-D map of galaxies and quasars found in the sample. An additional $225 \mathrm{deg}^{2}$ southern survey will be imaged repeatedly on varying timescales. Here we discuss both archival searches in the SDSS catalog (such as SDSS J24602.54+011318.8) and active searches with the SDSS instruments (such as for GRB 010222) for GRB afterglows and other transient objects.
\end{abstract}

\section{INTRODUCTION: THE SLOAN DIGITAL SKY SURVEY}

The SDSS is a project to image $10,000 \mathrm{deg}^{2}$ of the Northern Galactic Cap in five different filters $(u, g, r, i, z)$ to a depth of $r^{*} \sim 23$ and to perform followup spectroscopy of $10^{6}$ galaxies and $10^{5}$ quasars found in the photome- try $[13,16]$. The SDSS imaging survey is designed to be on an $A B_{v}$ system described in Fukugita et al. [2] where flat spectrum objects $\left(F_{\mathrm{V}} \propto \mathrm{v}^{0}\right)$ have zero colors. The magnitudes in this poster are quoted on the preliminary $u^{*}, g^{*}, r^{*}, i^{*}, z^{*}$ system which may differ by at most a few percent from the system of Fukugita et al. [2]. The spectroscopic survey will map galaxies to a depth of $z \sim 0.2$,

CP662, Gamma-Ray Burst and Afterglow Astronomy 2001: A Workshop Celebrating the First Year of the HETE Mission, edited by G. R. Ricker and R. K. Vanderspek

(C) 2003 American Institute of Physics 0-7354-0122-5/03/\$20.00 
luminous red galaxies to $z \sim 0.5$, and quasars as distant as $z \sim 6.5$.

The survey uses two dedicated telescopes, the automated $0.5 \mathrm{~m}$ "Photometric Telescope" (PT) which monitors extinction and provides calibration information, and the $2.5 \mathrm{~m}$ survey telescope used for the main imaging and spectroscopic surveys, both of which are located at Apache Point Observatory (APO) in Sunspot, New Mexico. The PT is an $\mathrm{f} / 8.8,0.5 \mathrm{~m}$ telescope equipped with ugriz filters on a filter wheel. The single SITe $2048 \times$ 2048 CCD camera has a $41.5^{\prime} \times 41.5^{\prime}$ field of view. A normal exposure of $300 \mathrm{sec}$ in $u$ and $120 \mathrm{sec}$ each in $g$, $r, i$, and $z$ reaches limiting $10 \sigma$ magnitudes of approximately $17.0,16.0,15.5,15.0$, and 15.0, although images as deep as $r^{*} \sim 19$ are regularly attained with longer exposures. Operation of the PT is fully automated (short of opening and closing the dome due to safety concerns) with targets for observation chosen from a queue based on assigned priorities and observability.

The $2.5 \mathrm{~m}$ survey telescope is an $\mathrm{f} / 5,3^{\circ}$ field of view telescope designed and constructed for the SDSS. The telescope has two interchangeable instruments, a 54 CCD imaging camera and a fiber-fed spectrograph capable of simultaneously observing 640 spectra. The imaging camera [3] includes an array of $302048 \times 2048$ CCDs in six columns of five CCDs each, one CCD for each of the 5 filters. The camera operates in a drift scan mode, scanning the sky in great circles at sidereal rate. Astronomical objects are imaged sequentially in the order $r, i, u, z, g$ for 53.9 seconds in each filter. Because of the gaps between columns the telescope must observe a second such interleaved strip to make a complete stripe. The survey is designed to reach $u^{*} g^{*} r^{*} i^{*} z^{*}$ magnitude limits of 22.3, 23.3, 23.1, 22.3, and 20.8. Galaxies, quasars, and other targets are selected from this imaging data for the spectroscopic survey.

\section{ARCHIVAL SEARCHES IN THE SDSS CATALOG}

Although the SDSS is not designed to search for GRB afterglows or variable objects of any sort, the multicolor observations make it possible to search for rare objects with non-stellar spectral energy distributions (SEDs) $[14,15,13,12,7]$. GRB afterglows are observed to have power law SEDs of the form $F_{v} \propto v^{\beta}$ with typical values of $\beta \sim-1$. Our initial studies have shown that such objects are rare but do exist in the SDSS catalog. Unfortunately reddened quasars have similar colors and can only be ruled out with temporal information. However several hundred $d \mathrm{~g}^{2}$ have already been scanned at least twice and the $225 \mathrm{deg}^{2}$ of the southern survey will be scanned dozens of times. Much work is currently under-

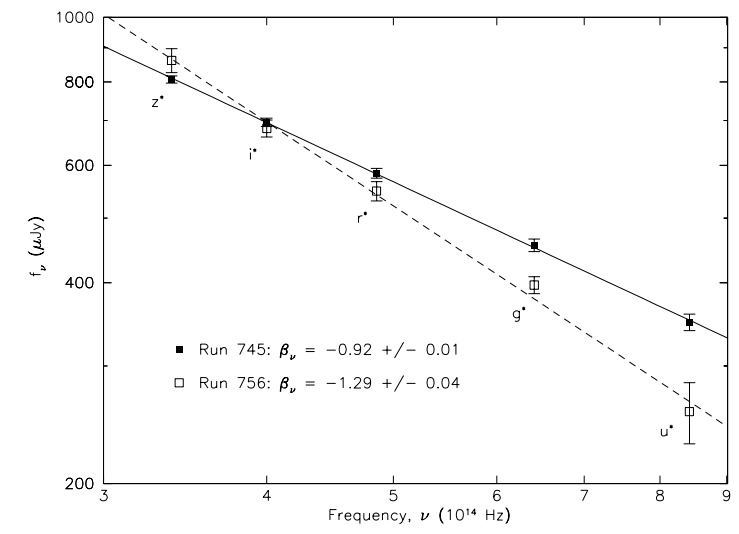

FIGURE 1. Spectral energy distribution of SDSS J124602.54+011318.8

way to find $\mathrm{SNe}$ and other variable objects in the southern survey within a day or less of observation.

Many objects which lie outside the stellar locus are automatically targeted for observation in the spectroscopic survey. In Vanden Berk et al. [15] we have used spectrophotometric magnitudes derived from these observations to provide temporal information and search for varying objects such as SNe. We have found one optical transient (OT) which shares many of its properties with GRB afterglows (see Vanden Berk et al. [14]). This transient was imaged at three separate epochs as part of the SDSS imaging survey and the spectrum was measured twice. A bright point source was observed in images separated by 2 days; all other SDSS and archival plate images show a galaxy 2.5 magnitudes fainter with no evidence of variability. ROTSE-I observations [5] from 50 days before to 50 days after show that the OT was not significantly brighter than the SDSS detection, indicating the SDSS detected the OT at its peak. The SED (Figure 1) is quite similar to that of GRB afterglows. While it is possible that this OT is a very unusual AGN, it seems much more likely that it is an "orphan afterglow" - a GRB afterglow without any observed $\gamma$ - ray flux [14].

\section{ACTIVE SEARCHES WITH THE SDSS TELESCOPES}

In certain rare cases when conditions do not allow normal survey operations the SDSS observers have been able to manually follow up some GRB triggers, including GRB010222 with the $2.5 \mathrm{~m}$ and $0.5 \mathrm{~m}$ PT telescopes [9], and more recently GRB010921 [8] and GRB011019 with the PT alone. The $2.5 \mathrm{~m}$ survey telescope observations of GRB010222 show the value of early multicolor observations. In addition to the measurement of the $g^{*} r^{*} i^{*} z^{*}$ spectral slope $\left(F_{v} \propto v^{-0.90 \pm 0.03}\right.$ for GRB010222), the ob- 


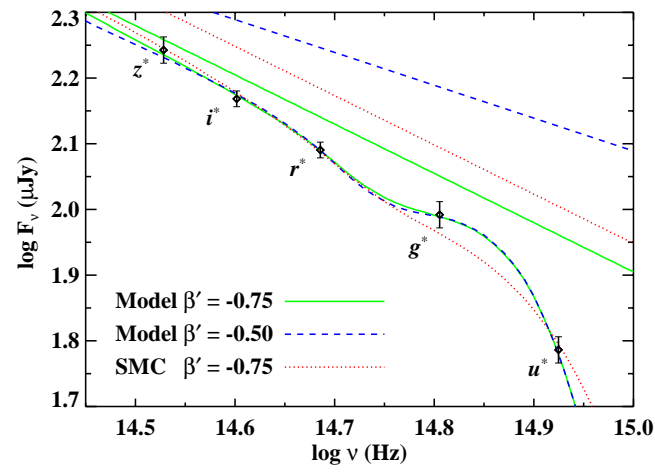

FIGURE 2. Extinction model fits to the $2.5 \mathrm{~m}$ multiband observations.

served break to a steeper slope in $u^{*}$ (Figure 2) was not predicted or seen in spectra, and likely indicate extinction at the source, possibly in a star forming region modified by the GRB or its progenitor. GRB010921 was observed under partially cloudy conditions approximately 1 and 2 days after the HETE-I trigger, and was seen to fade significantly from the first night $(r \approx 19.6)$ to the second. We have not yet detected a candidate object for GRB011019.

The above manual PT followup attempts have all suffered due to long delays between GRB detection and PT observation - the afterglow is often near the limiting magnitude of a $0.5 \mathrm{~m}$ telescope by the time PT observations can be scheduled. With trivial modifications to the PT's scheduling code, GCN alert positions could be inserted in the target queue, resulting in observations starting on average 10 minutes after the trigger. Additional modifications could reduce the average response time considerably. Existing PT image processing code may allow for detection of transients within minutes, allowing early spectroscopic followup on non-SDSS telescopes such as the ARC $3.5 \mathrm{~m}$ telescope at Apache Point. We are still in the early stages of studying whether a GRB followup mission could be funded and incorporated without any impact on the PT's primary mission as part of SDSS operations.

\section{CONCLUSIONS}

Our searches for GRB afterglows and other transients in the SDSS have already made many valuable discoveries, including what may be the first orphan afterglow and a strong indication of dust extinction in an afterglow's environment. Additional detections of transients with properties similar to GRB afterglows can test the beaming hypothesis and provide the first measurements of the properties of so called "orphan afterglows." Early near simultaneous observations of GRB afterglows spanning the Sloan ugriz filters, especially in the bluer filters, are nearly non-existent, yet these observations (along with $\mathrm{x}$ ray, near-IR, radio, and submillimeter observations) are needed to test the current ultra-relativistic external shock model of GRB afterglows, and to provide information about extinction of burst afterglows.

\section{ACKNOWLEDGMENTS}

The Sloan Digital Sky Survey (SDSS) is a joint project of The University of Chicago, Fermilab, the Institute for Advanced Study, the Japan Participation Group, The Johns Hopkins University, the Max-Planck-Institute for Astronomy (MPIA), the Max-Planck-Institute for Astrophysics (MPA), New Mexico State University, Princeton University, the United States Naval Observatory, and the University of Washington. Apache Point Observatory, site of the SDSS telescopes, is operated by the Astrophysical Research Consortium (ARC).

Funding for the project has been provided by the Alfred P. Sloan Foundation, the SDSS member institutions, the National Aeronautics and Space Administration, the National Science Foundation, the U.S. Department of Energy, Monbusho, and the Max Planck Society. The SDSS Web site is http://www.sdss.org/.

\section{REFERENCES}

1. Djorgovski, S. G. 2001, in Gamma-Ray Bursts in the Afterglow Era: 2nd Workshop, Proc. ESO Astroph. Symp., ed. N. Masetti et al., (Berlin: Springer Verlag), in press, (astro-ph/0107535)

2. Fukugita, M., Ichikawa, T., Gunn, J. E., Doi, M., Shimasaku, K., \& Schneider, D. P. 1996, AJ, 111, 1748

3. Gunn, J. E. et al. 1998, AJ, 116, 3040

4. Henden, A. 2001a, GCN Circ. 961

5. Kehoe, R., et al. 2001, in Supernovae and Gamma-Ray Bursts: The Greatest Explosions since the Big Bang, ed. M. Livio, N. Panagia, \& K. Sahu (Cambridge: Cambridge Univ. Press), 47

6. Kennicutt, R. C. 1998, ARA\&A, 36, 189

7. Krisciunas, K., Margon, B., \& Szkody, P. 1998, PASP, 110 , 1342

8. Lamb, D. Q., et al. 2001, GCN Circ. 1125

9. Lee, B. C., et al. 2001, ApJ, 561, 183 (astro-ph/0104201)

10. McDowell, J., Kilgard, R., Garnavich, P. M., Stanek, K. Z., \& Jha, S. 2001, GCN Circ. 963

11. Reichart, D. E. 2001, ApJ, 553, 235 (astro-ph/9912368)

12. Rhoads, J. E. 2001, ApJ, in press, (astro-ph/0008461)

13. Stoughton, C., et al. 2001, AJ, accepted

14. Vanden Berk, D. E., et al. 2001a, in preparation

15. Vanden Berk, D. E., et al. 2001b, in preparation

16. York, D. G. et al. 2000, AJ, 120, 1579 